\section{LA TASA DE DESCUENTO EN PAÍSES EMERGENTES APLICACIÓN AL CASO COLOMBIANO}

FECHA DE RECEPCIÓN: 5 de mayo FECHA DE APROBACIÓN: 29 de julio Pp. 120-135

\section{THE DISEOUNT RATE IN EMEREIWG GOUNTRIES- APPLIGATION OF THE GOLOMBIAN GASE}

\author{
Jairo Humberto Sánchez Segura*
}

* Administrador de Empresas, Universidad EAN; Especialista en Planeación y Desarrollo Regional, CIDER; Especialista en Finanzas; Magíster en Finanzas, Universidad de los Andes. Experiencia docente en el Instituto de Postgrados de la Universidad de la Sabana y Facultad de Administración Universidad EAN. Asesor administrativo y financiero de programas de gobierno (Presidencia de la República), Consultor administrativo y financiero en programas de cooperación internacional con la Unión Europea en Colombia, Gerente Administrativo y Financiero de compañias del sector real como MTM Ltda. y Marroquinera Mario Hernández S. A., Tesorero Universidad EAN. Actualmente Docente de la Facultad de Postgrados de la Universidad EAN. 


\section{RESUMEN}

$L$ a evaluación financiera se constituye en parte fundamental para determinar la viabilidad de los proyectos. En este proceso, el costo de capital se constituye en herramienta básica para medir no solo el retorno esperado, sino el riesgo percibido por los accionistas. A una mayor tasa esperada de retorno del capital, los proyectos serán más exigentes y requerirán mayores tasas de rentabilidad que recuperen la inversión y generen el retorno esperado por quienes asumen el riesgo de la financiación.

Este artículo presenta dos modelos financieros utilizados para el cálculo del costo de capital y los ajustes necesarios para calcular dicho costo cuando se trata de inversiones en paises emergentes. Parten ambos del modelo CAPM (Capital Assets Pricing Model) o modelo de precios de activos de capital y desarrolla los ajustes necesarios para calcular el mayor riesgo percibido en países emergentes que finalmente se refleja en la tasa de descuento. Se aplicarán ambos modelos al caso colombiano para determinar el costo de capital en inversiones efectuadas en su territorio.

\section{ABSTRACT}

The financial evaluation is considered a fundamental issue to determine the feasibility of projects. In this process, the capital cost becomes a basic tool to measure not only the expected return, but also the risk as seen by shareholders. To an expected high capital return rate, projects would become more demanding and would require higher profitability rates that recover investment and generate the expected return for those who assume the risk of financing.

In this paper, two financial models are presented as used to calculate capital cost and to make the necessary adjustments to also calculate this cost when investing in emerging countries. Both models are based on CAPM model (capital assets pricing model) or capital active pricing model and develop the necessary adaptations to calculate the highest risk observed in emerging countries that is finally reflected in a discount rate. Both models are applied to the Colombian case to determine the capital cost of investments made in this country.

\section{RESUMÉÉ}

L'évaluation financière est constituée fondamentalement pour déterminer la viabilité des projets. Dans ce processus, le coût du capital est un outil de base pour mesurer non seulement le retour sur investissement, mais également le risque perçu par les actionnaires. $A$ un plus grand taux de retour sur investissement du capital, les projets seront plus exigeants et requerront de plus grands taux de rentabilité qui récupèreront l'investissement et produiront le retour sur investissement nécessaire pour que le risque de financement soit assuré. Cet article présente deux modèles financiers utilisés pour le calcul du coût du capital et des ajustements nécessaires pour calculer ce coût quand il s'agit d'investissements dans des pays émergents. Ces deux modèles financiers s'inspirent du modèle CAPM (Capital Assets Pricing Model) ou modèle de prix d'actifs de capital et développe les ajustements nécessaires pour calculer le plus grand risque perçu dans des pays émergents qui se reflète finalement dans le taux de remise. On appliquera les deux modèles au cas colombien pour déterminer le coût du capital lors d' investissements effectués sur ce territoire.

\section{Palabras claves}

Costo de Capital

Rendimiento

Riesgo

Tasa libre de riesgo

CAPM (Modelo de precios

de activos de capital-Capital Assets Pricing Model) $\rightarrow \quad$ Key words

Capital cost

Economy of use

Risk

Risk free rate

CAPM (capital active

pricing model)

\section{$\Rightarrow \quad$ Mots clefs}

Cout du capital

Rendement

Risque

Taux libre de risque

CAPM (Modèle de prix

d'actifs capital-important

d'Assets Pricing Model) 


\section{INTRODUCCIÓN}

\section{E} tasa de descuento en un país emergente (caso Colombia), aplicado a diferentes sectores, ajustado en particular a las compañías dada su razón deuda/patrimonio (D/P) y se abordan en este proceso las dificultades que se tienen al aplicar para países emergentes modelos teóricos (de amplia utilización en mercados desarrollados); el procedimiento técnico utilizado; y especialmente, el tipo, tratamiento y calidad histórica de la información que se requiere y obtiene del mercado de capitales en el caso colombiano.

En particular se pretende dejar explícita la metodología utilizada para su aplicación en otras empresas del sector real de la economía y para otros países teniendo en cuenta las externalidades que se pueden presentar en su desarrollo.

El modelo básico utilizado es el Capital Assets Pricing Model (CAPM) o Modelo de Precios de Activos de Capital e incluye las modificaciones sugeridas por algunos autores para su aplicación en el cálculo de la tasa de descuento para inversiones en países emergentes. En estos mercados se presentan riesgos adicionales desencadenados por la exposición de las inversiones a factores propios de estas economías que se pueden agrupar en políticos, comerciales y de tipo de cambio (Stephen, et al., 1996) .

En el riesgo político las inversiones están expuestas a la incertidumbre por los cambios en la normatividad legal vigente, así como en las políticas de Estado. El comercial es inherente a la actividad propia del negocio en el mercado local y tiene que ver con su profundidad, el desarrollo de la competencia y especialmente con las barreras de entrada y salida, lo que incrementa la volatilidad en la rentabilidad de los activos. En el riesgo de tipo de cambio, las inversiones y los flujos monetarios internacionales están expuestos a los precios diferenciales de las monedas en las cuales se transan y estos dependen del desarrollo, grado de interrelación y coyuntura particular de las economías que los comparten.

Es claro entonces, que los riesgos descritos, sobre los cuales las empresas no tienen control, se reflejen en una mayor tasa que el inversionista exigirá por su exposición. Dicha tasa es el resultado de la medición del riesgo descrito y su incorporación.

Los resultados para el cálculo de la tasa de descuento para inversiones en Colombia (sector procesamiento de alimentos, razón deuda patrimonio de una compañía del sector tomada como ejemplo) fue de 11,97\% (modelo CAPM ajustado, Damodaran) y de 11,29\% (modelo de Godfrey y Espinosa). Los modelos reflejan una aproximación a la rentabilidad exigida por el inversionista y la decisión de utilizarla, depende finalmente de sus expectativas de comportamiento, de los diversos factores involucrados en el cálculo y del tipo de inversión en particular.

En la primera parte de este artículo se describen brevemente las aproximaciones teóricas de algunos autores respecto al cálculo de la tasa de descuento para países emergentes; en la segunda, se indica el sector seleccionado y los métodos que se aplicarán en el cálculo de la tasa de descuento; en la tercera parte se muestra la aplicación práctica de los métodos y análisis de los resultados obtenidos; en la última, se presentan las conclusiones. 


\section{REVISIÓN DE LA LITERATURA}

1 ara abordar el tema de inversiones en países emergentes, se considera un inversionista local, en una economía desarrollada, la cual tiene condiciones de mercado profundo, con amplia información y condiciones macroeconómicas de largo plazo estables. En estas circunstancias, realiza estimaciones de la tasa de retorno de su capital y toma decisiones acordes con el nivel de riesgo que enfrenta. Cuando el inversionista se traslada al mercado de capitales internacional y selecciona un país emergente, debe tener en cuenta una serie de factores diferenciales que modifican el riesgo, los cuales debe incluir en la estimación de la tasa de descuento requerida para evaluar su inversión en estas condiciones.

El capital invertido en una empresa proviene de dos fuentes, el capital propio y la deuda. El primero (Equity) es invertido por los accionistas de la empresa, mientras que la deuda, son recursos financieros que son facilitados por el sector financiero o inversionistas, a unas tasas y plazos que por sí reflejan el costo de oportunidad de esos recursos. Para el caso del capital propio, la tasa de descuento está sujeta a las condiciones de riesgo del mercado donde opera la inversión y son diversas las metodologías desarrolladas para su cálculo.

Una de las metodologías más reconocidas es el Capital Assets Pricing Model (CAPM) o Modelo de Valoración de Activos de Capital. Este fue propuesto por William Sharpe como un modelo del riesgo y rendimiento en un artículo de 1964. En este modelo se parte del supuesto que sólo el riesgo no diversificable (sistemático) debe ser medido y compensado. Expresa que el rendimiento esperado de un activo depende de tres factores: la tasa libre de riesgo (Rf), la tasa del mercado (Rm) y el beta (ß). La tasa de retorno del activo entonces vendría dada por:
La tasa libre de riesgo (Rf) representa la mínima rentabilidad que un inversionista espera al colocar su dinero en inversiones libres de riesgo (como podrían serlo los bonos del tesoro americano). El mercado de capital espera el retorno con una tasa Premium sobre la tasa libre de riesgo de activos de referencia (Bonos o notas del Tesoro Americano) para compensar a los inversionistas por el riesgo adicional involucrado en mantener dichas inversiones de capital. La diferencia entre paréntesis mide la rentabilidad en exceso que genera el mercado sobre la tasa libre de riesgo y es ampliada o disminuida en función del beta que mide la sensibilidad del activo a los cambios en el mercado.

Este modelo predice la relación que existe entre el riesgo y la rentabilidad de una inversión. El punto de fondo es que si bien hay un acuerdo generalizado sobre la tasa libre de riesgo, el análisis de la prima del mercado (Rm-Rf) tiene profundas diferencias, y más cuando se calcula en países con poca historia de sus mercados o cuando estos son muy volátiles (caso de países emergentes).

En esta perspectiva Damodaran (1999), cita varios modelos que tienen en cuenta el riesgo bajo la perspectiva anotada. El primer Modelo CAPM ya mencionado y comentado; el modelo Arbitrage Pricing Model (APM) que indica que inversiones con la misma exposición al riesgo de mercado tienen que negociarse al mismo precio (no hay arbitraje), para lo cual se incluyen en el modelo, betas calculados contra múltiples factores de riesgo de mercado. En el Multi-Factor Model, los betas se calculan contra múltiples y específicos factores macroeconómicos; y finalmente, el Proxy Model que asume que sobre muy largos períodos de tiempo, altos retornos sobre las inversiones deben ser la compensación por el alto riesgo del mercado.

$\mathbf{R a}=\mathbf{R f}+(\mathbf{R m}-\mathbf{R f}) * \mathbf{B}$ 
En los tres primeros modelos el retorno esperado de cualquier inversión estará dado por:

Retorno esperado: Tasa libre de riesgo $+b_{j} \sum_{j=1}^{i=k}$

(Prima Riesgo del mercado $\mathrm{j}$ ).

donde $b_{j}$ (beta) y la prima de riesgo del mercado corresponden al factor $\mathrm{j}$.

En el modelo simplificado del CAPM, la tasa libre de riesgo asume la de los títulos del tesoro americano (Tbonds por ejemplo) y la prima de riesgo es el exceso del mercado (índice de S\&P500 por ejemplo) sobre la tasa libre de riesgo. El beta puede tomarse de la base de datos disponible por Damodaran. Para el ajuste de esta tasa calculada en Estados Unidos, para países emergentes, se debe adicionar la prima de riesgo del país para el cual se va a calcular, (country equity risk premium o prima riesgo país sobre patrimonio).

El procedimiento es el de calcular la tasa en exceso del mercado de acciones en el país emergente sobre su tasa libre de riesgo (la de sus bonos soberanos o papeles de gobierno). La diferencia, sobre una base anual, entre los dos retornos es calculada y representa la tasa histórica de riesgo país. El resultado es el spread promedio sobre sus bonos soberanos (country default spread) que los inversionistas en acciones están exigiendo. El cálculo de la tasa de riesgo país es un paso intermedio para estimar la prima de riesgo país que se usa en los modelos de riesgo. Para efectuar este último cálculo se debe analizar la volatilidad del mercado de acciones (Equity) del país emergente relativo al mercado de sus bonos soberanos (country bond) y utilizarla para ajustar la tasa anterior.

\section{Prima sobre patrimonio riesgo país $=$ Country Default Spread * (Sequity / Scountry Bond)}

Otra aproximación es presentada por Erb, Harvey y Viskanta (1996), donde el riesgo país es el resultado de cinco diferentes cálculos de riesgo, cuatro de estos contenidos en la Guía Internacional de Riesgo País que incluye variable política, financiera, económica e índice de riesgo compuesto y una medida adicional relacionada con la calificación de crédito en el país del inversionista institucional, (este nivel de riesgo es dado por líderes de los principales bancos internacionales). El análisis del comportamiento de las diferentes variables puede dar luces sobre los componentes del riesgo para un país determinado e incluye un análisis profundo de diversas series de datos y sus correlaciones con factores del mercado como los indicados.

En un nuevo abordaje al cálculo del riesgo país, (Estrada, 2000), argumenta que la versión internacional del CAPM asume mercados muy integrados, lo que implica que activos con el mismo riesgo deben tener el mismo retorno esperado independientemente del mercado donde son transados. Hay evidencia que esto no es cierto para todos los mercados y su grado de integración es diferente. Trata de aproximarse a este cálculo primero confirmando que el retorno del mercado se basa en dos componentes, la tasa libre de riesgo y la prima de mercado. El primero es una compensación requerida por la pérdida esperada del poder de compra que es demandada incluso por un activo sin riesgo. El segundo componente es una compensación extra por enfrentar el riesgo y depende del activo considerado. Toma la perspectiva del inversionista localizado en Estados Unidos diversificado internacionalmente. La tasa libre de riesgo debe compensarlo por la expectativa de pérdida del poder de compra del dólar y el risk Premium (Prima sobre el riesgo de mercado) debe compensarlo por el riesgo de invertir en el portafolio de mercado en el mundo (cualquier país de que se trate). Estrada también plantea medir la prima del mercado mediante lo que denomina downside risk calculado como la razón entre la desviación semiestándar de los retornos con respecto a la media en el mercado (local), y la desviación semiestándar de los retornos con respecto a la media en el mercado mundial. La complejidad del modelo radica en identificar las variables que lo miden, especialmente las del mercado mundial que presumiría algún grado de correlación e integración entre todos los mercados. Una aproximación alternativa puede estar en las calificaciones de riesgo provistas por agencias calificadoras. El problema es que aplica para riesgo país pero no para el nivel de compañía.

En mercados emergentes, betas y el retorno de las acciones no están muy correlacionados. Godfrey y Espi-nosa (1996) argumentan que las firmas que invierten internacionalmente asumen riesgos de incertidumbre como las tasas de impuestos, regulaciones y normas legales, régimen de cambio, políticas macroeconómicas; todo ello genera volatilidad en los negocios. Proponen ajustar el CAPM de dos maneras: primero adicionando a la tasa libre de riesgo, el spread entre el retorno de un bono soberano del mercado emergente y el retorno de un bono comparable 
en los Estados Unidos. Segundo, usando un beta ajustado definido como el $60 \%$ de la razón entre la desviación estándar de los retornos en el mercado emergente y la desviación estándar de los retornos en el mercado de los Estados Unidos.

$$
\mathbf{R a}=\mathbf{R f}, \mathbf{U S D}+\text { Credit Spreadi }+(\mathbf{R m}-\mathbf{R f}) * \mathbf{B a} \mathbf{i}
$$

$\mathbf{R}_{\mathrm{f}}$, USD: Tasa de interés libre de riesgo en Estados Unidos

Credit Spreadi: Spread que refleja la calidad de crédito país i. Mide la diferencia entre los bonos soberanos en moneda local y los denominados en moneda extranjera, recogiendo así la percepción del riesgo país por el mercado internacional.

( $\mathbf{R m}-\mathbf{R f})$ : Risk Premiun, tasa de retorno por encima del mercado en país desarrollado.

Bai: Beta ajustado para el país i. Definido como el $60 \%$ de la razón $\sigma i$ / $\sigma \mathrm{m}$ equivalente a volatilidad del mercado foráneo/volatilidad del mercado en los Estados Unidos.

La aproximación de Godfrey y Espinosa no tiene en cuenta la tasa para un proyecto específico en un país emergente. Por último, es importante considerar la situación de empresas ubicadas en negocios que no se comportan de acuerdo con el libre mercado, sino en situaciones de mercados regulados como lo es el caso de servicios públicos, entre otros. Hay evidencia acerca de cómo la selección de un régimen regulado afecta el nivel de riesgo de los accionistas de una compañía. Alexander, Mayer y Weeds (1996), describen los diferentes tipos de regulaciones en las que se encuentran:

Precio (Price Cap); ingresos (Revenue Cap Regulation), tienen sentido en el caso de altos costos fijos (se incluye un cargo fijo en la tarifa), pero genera problemas por alta fluctuación de la demanda; tasa de retorno (Rateof-Return Regulation), asegura la tasa de retorno del inversionista y los precios se ajustan de ser necesario para mantenerla. El estudio concluye que los incentivos de alta potencia están relacionados con mayor riesgo sistémico (inducido por mayores niveles de regulación) mientras que los incentivos de baja potencia implican bajo riesgo de mercado (mayor libertad de mercado, bajo nivel regulatorio). Por lo anterior, es importante tener en cuenta los elementos de riesgo implícito en los sistemas regulatorios y cómo estos aumentan el riesgo sistemático, requiriendo en compensación mayores tasas de retorno dado el incremento en el costo de capital.

\section{CASO COLOMBIANO Y SELECGIÓN DE LOS MODELOS}

L. a selección del país obedece al interés del autor por conocer los ajustes en el modelo CAPM para un país emergente como lo es Colombia, que forma parte del grupo de países latinoamericanos, zona que ha tenido momentos complejos desde el punto de vista económico y político y dentro del cual Colombia ha presentado estabilidad macroeconómica, de política fiscal y no ha entrado en default de su deuda externa.

El primer modelo que se aplicará para calcular el costo de capital en Colombia será el desarrollado por Damoradan para la estimación del Country Risk Premium (Prima de riesgo país).
Se considera que para el cálculo de la tasa de descuento de una compañía privada, el modelo más apropiado es el CAPM, no sólo por el sustento teórico que lo respalda, su amplia aceptación y uso generalizado en este campo, sino porque puede ajustarse dadas las condiciones de mercado en el cual se aplique, permitiendo un modelaje financiero que se acerca a la tasa apropiada de descuento en economías de países emergentes. El modelo original puede ser modificado para ajustarse a economías diferentes al mercado de los Estados Unidos, considerado un mercado maduro, con amplias bases históricas que proveen información sobre el comportamiento de los diferentes activos del mercado. La aproximación estándar para estimar el Equity Risk Premium descansa en el uso de los retornos históricos con la diferencia entre los retornos 
anuales sobre las acciones y los bonos sobre un largo período de tiempo. En países emergentes las bases de datos históricas tienden a ser limitadas y ruidosas (en términos estadísticos se refiere a shocks temporales que afectan la serie). El profesor Damodaran sugiere procedimientos para la estimación del Equity Risk Premium para mercados emergentes utilizando la tasa base de acciones transadas (base Equity Premium) y la tasa de riesgo país.

El segundo modelo seleccionado es el presentado por Godfrey y Espinosa, quienes argumentan que los tres mayores tipos de riesgo que afectan las inversiones en países desarrollados son el político (riesgo soberano), el comercial y el riesgo de moneda. El primero puede ser determinado observando el spread de la tasa sobre los bonos soberanos denominados en una reserva de moneda común como es el dólar. El riesgo del negocio o comercial puede ser medido comparando la volatilidad del mercado de acciones local con la volatilidad del mercado de acciones en Estados Unidos. El riesgo de moneda puede ser explicado realizando el análisis en dólares de Estados Unidos (o moneda local), esto es convirtiendo los flujos de caja de moneda local (dólares), a un apropiado tipo de cambio y entonces descontando esos flujos en dólares a la tasa apropiada de riesgo en dólares.

Los dos modelos se aplicarán para Colombia en algunos sectores indicados, por cuanto permiten medir el riesgo que se presenta en el país y las modificaciones argumentadas por los autores pueden realizarse con la información disponible, aun con las dificultades anotadas en países emergentes $\mathrm{y}$ de las cuales Colombia no es ajena. El modelo ajustado de Damodaran permite adicionalmente incluir el beta para dife-

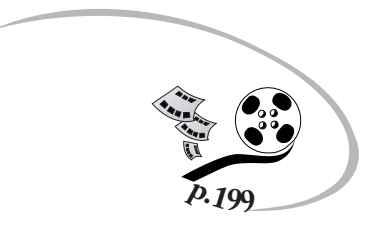
rentes sectores ajustando la tasa Premium de riesgo país a los de diferentes sectores para los que Damodaran ha calculado el beta.

En el caso colombiano es importante indicar que el índice IGBC de la Bolsa de Valores no incluye un amplio espectro de firmas y de las que lo hacen, la concentración de propiedad accionaria es muy alta dejando un porcentaje muy bajo para ser negociado en el mercado.

\subsection{ANÁLISIS}

\subsubsection{Modelo 1}

En la aplicación del primer modelo, el primer paso está en estimar la tasa libre de riesgo que será la de los bonos americanos (tabla 1) y se seleccionaron los T-bonds del periodo 1928-2009, promedio geométrico. Los cálculos están ya elaborados en la página web del profesor Damodaran.

Tabla 1. Tasa libre de riesgo Estados Unidos

MARKET RISK PREMIUM

Arithmetic Average

\begin{tabular}{|c|c|c|c|c|c|c|c|}
\hline PERIOD & T.Bills & T.Bonds & PERIOD & \multicolumn{2}{|c|}{ Risk Premium } & \multicolumn{2}{|c|}{ Standard Error } \\
\hline & & & & Stocks-T.Bills & Stocks-T.Bonds & Stocks-T.Bills & Stocks-T.Bonds \\
\hline 1928-2009 & $3,74 \%$ & $5,24 \%$ & 1928-2009 & $7,53 \%$ & $6,03 \%$ & $2,276 \%$ & $2,405 \%$ \\
\hline 1960-2009 & $5,33 \%$ & $7,03 \%$ & 1960-2009 & $5,48 \%$ & 3,78 & 2,4225 & $2,709 \%$ \\
\hline \multirow[t]{2}{*}{ 2000-2009 } & $2,74 \%$ & $6,62 \%$ & $2000-2009$ & $-1,59 \%$ & $-5,47 \%$ & $6,729 \%$ & $9,224 \%$ \\
\hline & & & & \multicolumn{2}{|c|}{ Risk Premium } & & \\
\hline $\begin{array}{c}\text { Geometric } \\
\text { Average }\end{array}$ & T.Bills & T. Bonds & & Stocks-T.Bills & Stocks-T.Bonds & & \\
\hline $1928-2009$ & $3,70 \%$ & $4,97 \%$ & 1928-2009 & $5,56 \%$ & $4,29 \%$ & & \\
\hline 1960-2009 & $5,29 \%$ & $6,64 \%$ & 1960-2009 & $4,09 \%$ & $2,74 \%$ & & \\
\hline 2000-2009 & $2,72 \%$ & $6,26 \%$ & 2000-2009 & $-3,68 \%$ & $-7,22 \%$ & & \\
\hline
\end{tabular}

Fuente. Damodaran on line (página del profesor Damodaran, NYU). Updated data, historical returns on stocks Bonds and Bills-United States. Disponible en: http//pages.stern.nyu.edu/-adamodar/.Consult:25 de febrero de 2010. 
El segundo paso es calcular la prima de riesgo país a partir del riesgo de sus papeles soberanos, calculados sobre el spread entre estos y los papeles libres de riesgo en mercados maduros (como es el caso de los bonos del tesoro americano).

\section{Tabla 2. Embi Spread Colombia}

\begin{tabular}{|l|l|l|}
\hline \multicolumn{2}{|c|}{} & \multicolumn{1}{c|}{ Período } \\
\hline 1 año & $3,126 \%$ & 2 Enero-09/ 3 Enero-10 \\
\hline 2 años & $3,081 \%$ & 4 Enero-08/ 3 Enero-10 \\
\hline 3 años & $2,610 \%$ & 4 Enero-07/ 3 Enero-10 \\
\hline 5 años & $2,589 \%$ & 4 Enero-05/ 3 Enero-10 \\
\hline 7 años & $3,159 \%$ & 3 Enero-03/ 3 Enero-10 \\
\hline 10 años & $4,155 \%$ & 4 Enero-00/3 Enero-10 \\
\hline
\end{tabular}

Fuente. JP Morgan, servicio de suscripción, fecha: 25 febrero 2010.

Se tomará la tasa de un año la cual concuerda con la desviación estándar más baja. La tasa obtenida debe ajustarse por el siguiente producto:

Country Equity Risk Premium = Country Default Spread X ( $\sigma$ EQUITY / $\sigma$ COUNTRY BOND)

бEQUITY: Desviación estándar de los rendimientos de índice accionario del país sobre el que se quiere medir el Equity Risk Premium.

бCOUNTRY BOND: Desviación estándar del EMBI que mide el spread de los bonos soberanos del país frente a la tasa libre de riesgo en mercados maduros (bonos del tesoro americano).
Para el cálculo de la desviación estándar de inversiones de patrimonio en Colombia ( $\sigma$ EQUITY) se ha tomado como base el Índice General de la Bolsa de Valores Colombia (IGBC), período 7 de marzo de 2001 a 3 de febrero de 2010. Se realizó ajuste del índice a dólar de los Estados Unidos a la TRM de cada fecha y la rentabilidad se ha medido con base en el interés compuesto continuo diario. El resultado es el cálculo de la desviación estándar diaria (equivalente anualizada).

Tabla 3. Retorno promedio y desviación estándar del IGBC, Colombia

\begin{tabular}{|l|r|r|}
\hline Período: 7 de marzo 2001 al 3 de febrero 2010 & \multicolumn{1}{|c|}{ Valor USD } & \multicolumn{1}{c|}{ Valor COP } \\
\hline Retorno promedio & $0,13 \%$ & $0,12 \%$ \\
\hline Desviación estándar diaria & $1,63 \%$ & $1,49 \%$ \\
\hline Desviación estándar anualizada & $25,94 \%$ & $23,57 \%$ \\
\hline
\end{tabular}

Fuente. Grupo AVAL, disponible en: http//WWW.grupoaval.com/portal/page?_pageid=33,115460184\&_schema= PORTAL. Consulta: Febrero 25 de 2010.

Para el cálculo de la desviación estándar del EMBI se tomará la serie EMBI Colombia de la tabla 2 y se calculará la desviación estándar diaria (anualizada) (tabla 4). El período es el mismo de la Tabla 2 para un año. 
Tabla 4. Desviación estándar EMBI SPREAD Colombia

\begin{tabular}{|l|l|l|}
\hline & Diaria & \multicolumn{1}{|c|}{$\begin{array}{c}\text { Desviación } \\
\text { estándar año }\end{array}$} \\
\hline 1 año & $1,061 \%$ & $16,849 \%$ \\
\hline 2 años & $1,232 \%$ & $19,562 \%$ \\
\hline 3 años & $1,240 \%$ & $19,691 \%$ \\
\hline 5 años & $1,088 \%$ & $17,275 \%$ \\
\hline 7 años & $1,384 \%$ & $21,969 \%$ \\
\hline 10 años & $2,064 \%$ & $32,758 \%$ \\
\hline
\end{tabular}

Fuente. JP Morgan, servicio de suscripción, fecha: 25 febrero 2010.

Si se asume que la exposición de una compañía al riesgo país es proporcional a su exposición a los otros riesgos del mercado que es calculado por el beta, entonces el beta afectaría directamente ambas tasas. Si se considera que afecta de manera diferenciada cada tasa de riesgo, entonces debería aplicarse el beta para la prima de riesgo en mercado maduro (USA) y un $\lambda$ (lamda) que afecte la tasa de riesgo país de acuerdo con criterios como el porcentaje de ventas locales y al exterior, valor ingresos en moneda local y en dólares, etc. Para Colombia asumimos que los proyectos se desarrollan en el país y tendrán $100 \%$ de riesgo país.

\section{Expected Return $=\mathbf{R f}+$ Beta $($ Mature Equity Risk Premium) $+\lambda$ (County Risk Premium)}

El cálculo completo de la tasa de riesgo país, utilizando los resultados anteriores se describe en la Tabla 5, la cual incluye la siguiente operación:

\section{Country Default Spread X (סEQUITY/OCOUNTRY BOND)}

Tabla 5. Prima de riesgo país (Colombia)

\section{COUNTRY RISK PREMIUM}

\section{Concepto}

Country Default Spread (Col)

Equity Index Standard Deviation (IGBC COL)

EMBI Col Index Standard Deviation (COL)

Desviación estándar relativa

Country Risk premium
Tasa

$\begin{array}{cl}3,13 \% & 1 \text { years average EMBI: JP Morgan } \\ 25,94 \% & \\ 16,85 \% & 1,54 \\ 1,54 & \text { Volatilidad relativa Equity/Bond } \\ 4,81 \% & =3,13 \% \times(25,94 \% / 16,85 \%)\end{array}$

Ahora se estimará el beta correspondiente para calcular la tasa de descuento y aplicar el modelo CAPM completo. El beta se tomó de la página web del profesor Damodaran y se seleccionó en la tabla de betas para países emergentes el correspondiente a compañías procesadoras de alimentos, tal como se señala en la tabla 6 . 
Tabla 6. Estimación del Beta

\begin{tabular}{|c|c|c|c|c|c|}
\hline Concepto & Industria & Fuente & $\mathbf{b}_{u}$ & $\mathbf{\%}$ & Weighted Factor \\
\hline $\begin{array}{c}\text { Unievered } \\
\text { Beta/ } \\
\text { Emerging market }\end{array}$ & $\begin{array}{c}\text { Food } \\
\text { Processing }\end{array}$ & $\begin{array}{c}\text { Damodaran/corregido } \\
\text { por caja }\end{array}$ & 0,473 & $100,0 \%$ & $47,3 \%$ \\
\hline & & \multicolumn{3}{|l|}{ Weighted Average $b_{u}$} & 0,473 \\
\hline
\end{tabular}

Fuente. Damodaran on Line. Levered and unievered betas by industry. Disponible en http//pages.stern.nyu.edu/adamodar/.Consulta; 25 de febrero de 2010.

Para ajustar el beta utilizamos la relación deuda/equity que tenía una compañía del sector alimentos al cierre del 31 de diciembre de 2008 (Tabla 7), información disponible en la Superintendencia de Sociedades ${ }^{1}$.

Tabla 7. Relación deuda/patrimonio compañía sector alimentos

\begin{tabular}{|c|c|c|}
\hline & \multicolumn{2}{|c|}{$\begin{array}{l}\text { Cifras tomadas del informe de Supersociedades (SIREM) cierre de } 2008 \\
\$ \$ 000 \text { (Miles de pesos) }\end{array}$} \\
\hline C.P. & 251.882 & \\
\hline L.P. & 42.196 .050 & \\
\hline DEUDA & 42.447 .932 & 20,83 \\
\hline PATRIMONIO & 161.345 .516 & 79,17 \\
\hline CAPITAL & 203.793 .448 & \\
\hline RAZÓN D/E & $26,31 \%$ & \\
\hline
\end{tabular}

Fuente. Superintendencia de Sociedades; disponible en:http://sirem.supersociedades.gov.co/SIREM/index.jsp.Consulta: 26 de febrero de 2010.

Ahora se puede aplicar el CAPM completo dado que se cuenta con todos los elementos previamente identificados. En la siguiente tabla se detalla este ejercicio:

$$
\mathbf{R a}=\mathbf{R}_{\mathrm{f}} \mathbf{U S D}+\text { Credit }_{\text {Spread }_{\mathrm{i}}}+(\mathbf{R m}-\mathbf{R f}) * \mathbf{B}_{\mathrm{ai}}
$$

Tabla 8. Cálculo de la tasa de descuento para compañía del sector alimentos

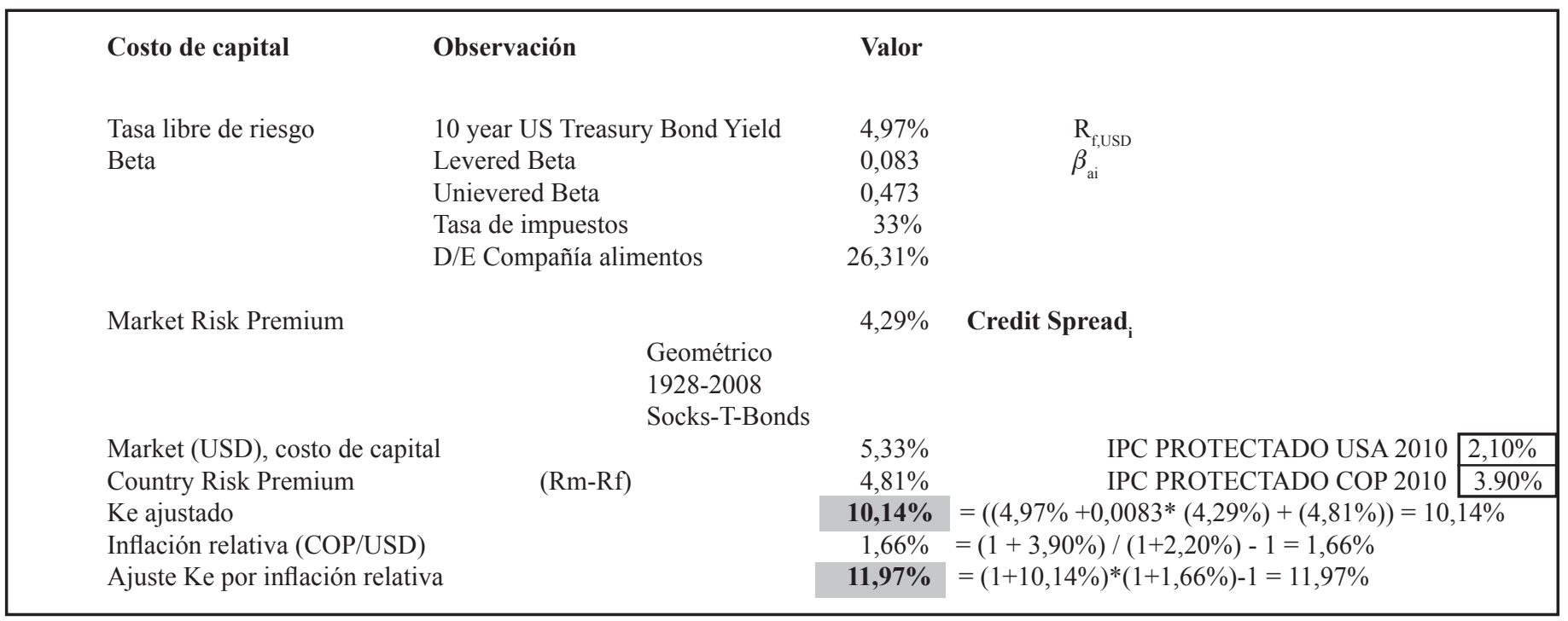

Fuente. Inflaciones estimadas: Bloomberg, servicio por suscripción. Consulta: marzo 2 de 2010.

\footnotetext{
${ }^{1}$ Información financiera compañía sector alimentos, Superintendencia de Sociedades, Colombia. Disponible en http://sirem.supersociedades.gov.co/SIREM/index. jsp. Consulta: 25 de febrero de 2010.
} 
El costo del Equity hallado en dólares, equivalente en moneda local, se obtiene efectuando el ajuste con la tasa de inflación relativa $(1+\mathrm{f}$ COP $) /(1+\mathrm{f}$ USD $)-1$.

Con la información construida y el modelo desarrollado, el costo de capital para una compañía del sector alimentos con razón $\mathrm{D} / \mathrm{P}$ de $26,31 \%$ sería de $13,02 \%$ para sus proyectos en Colombia.

\subsubsection{Modelo 2}

El argumento esgrimido por los autores Godfrey y Espinosa parte del modelo CAPM y sugiere para el cálculo de riesgo

Tabla 9. Desviación estándar del S\&P500, Estados Unidos

\begin{tabular}{|l|r|}
\hline \multicolumn{1}{|c|}{ Período: $\mathbf{7}$ de marzo de 2001 al 3 de febrero de 2010 } & \multicolumn{1}{c|}{ Valor USD } \\
\hline Desviación estándar diaria & $2,80 \%$ \\
\hline Desviación estándar anualizada & $44,40 \%$ \\
\hline
\end{tabular}

Fuente. Grupo AVAL, disponible en http//www.grupoaval.com/portal/page?_pageid=33,115460184\&_dad=portal\&_schema=PORTAL. Consulta ferbrero 25 de 2010.

Finalmente se pueden efectuar todos los cálculos del modelo tal como se detalla en la siguiente tabla:

Tabla 10. Modelo de Gofrey y Espinosa para el cálculo de la prima de riesgo país aplicado a Colombia

\begin{tabular}{|c|c|c|c|}
\hline \multicolumn{4}{|c|}{ 1. Cálculo del spread de colombia frente al mercado americano } \\
\hline \multicolumn{4}{|c|}{ La fuente JP Morgan tiene la serie de datos y está incluida en la hoja EMBI SPREAD. } \\
\hline \multicolumn{3}{|c|}{ EMBI SPREAD COLOMBIA } & \\
\hline \multicolumn{3}{|c|}{ PERIODO: } & \\
\hline $1 \mathrm{AÑO}$ & $3,126 \%$ & 2 ENERO-09 / 3 ENER - 1 & \\
\hline \multicolumn{4}{|c|}{ FUENTE: JP Morgan, servicio de suscripción, fecha:25 febrero 2010.} \\
\hline \multicolumn{4}{|c|}{ 2. Prima por la votalidad de inversiones en Colombia } \\
\hline \multicolumn{4}{|l|}{ BETA AJUSTADO= } \\
\hline \multicolumn{4}{|c|}{$\begin{array}{l}\text { 25,94\% Desviación Esándar IGBC Colombia medida en dólares } \\
44,40 \% \text { Desviación Estandar S\&P } 500 \text { ESTADOS UNIDOS } \\
\mathbf{0 , 5 8}\end{array}$} \\
\hline \multicolumn{4}{|c|}{$\begin{array}{l}\text { 3. Ajustes del Beta por interdependencia entre el riesgo de crédito y la volatilidad del } \\
\text { patrimonio. }\end{array}$} \\
\hline \multicolumn{4}{|c|}{$0,35$ (BETA = BETA AJUSTADO $\times 0.60)$} \\
\hline \multicolumn{4}{|l|}{ 4. Cálculo de la tasa de descuento. } \\
\hline \multicolumn{4}{|c|}{ k=rF us + Credit Spread + (60\% BETA xUS Equity Premium) } \\
\hline Rf US & $4,97 \%$ & \multirow{2}{*}{$\begin{array}{l}\text { IPC PROTECTADO USA } 2010 \\
\text { IPC PROTECTADO COPA } 2010\end{array}$} & $2,20 \%$ \\
\hline Credit Spread & $3,13 \%$ & & $3,90 \%$ \\
\hline BETA & $0,35 \%$ & & \\
\hline \multirow{2}{*}{$\begin{array}{l}\text { Us Equity premium } \\
\text { Costo Capital Colombia }\end{array}$} & $3,92 \%$ & & \\
\hline & $9,47 \%$ & \multicolumn{2}{|c|}{ Tasa en USD $=4,97 \%+3,13 \%+\left(0,35^{\star} 3,92 \%\right)=9,47 \%$} \\
\hline \multirow{2}{*}{ Inflación Relativa (COP/USD) } & $1,66 \%$ & & \\
\hline & $11,29 \%$ & Tasa en COP & \\
\hline
\end{tabular}

Fuente. A practical approach to calculating costs of equity for investments in emerging markets, Sephen Godfrey, Ramon Espinosa. Journal of Applied Corporate Finance. Bank of América, 1996. país, partir de la tasa libre de riesgo en un mercado maduro como es el de Estados Unidos y a este valor agregarle el spread (diferencia) entre los bonos soberanos del país emergente y el de los bonos del tesoro (para el mismo plazo), esto es, papeles de la misma naturaleza. El resultado debe ajustarse por un beta calculado como el $60 \%$ de la razón entre desviación estándar de la rentabilidad del patrimonio en el país emergente (IGBC para el caso colombiano, tabla 3) y la desviación estándar del mercado maduro (S\&P500 en Estados Unidos, tabla 9). 
Una de las diferencias con el modelo expresado por Damodaran está en el cálculo del Beta, que es estimado de acuerdo con la sensibilidad del rendimiento de las acciones de la empresa frente a los rendimientos de los activos del mercado. Hasta aquí los autores logran estimar el riesgo país para el caso de mercados diferentes al de Estados Unidos. La otra diferencia está en el ajuste que se hace a la prima de riesgo país. Mientras Damodaran utiliza el cociente entre la desviación del índice de la bolsa del país emergente y la desviación del Spread de sus bonos soberanos (datos del mismo país, IGBC y EMBI Colombia), Espinosa utiliza el cociente entre las desviaciones estándar de las bolsas de valores de los dos mercados (IGBC Colombia y S\&P500 para el caso presentado en este documento).
Para ajustar la exposición al riesgo de moneda, los autores sugieren convertir los flujos de caja de la compañía a dólares y con ello es factible utilizar la tasa encontrada por el modelo. El resultado es de aplicación directa en el país emergente con flujos de caja en su moneda para el modelo de Damodaran, y de ajuste en el flujo de caja, trasladando sus flujos a dólares y utilizando la tasa calculada en el modelo. Una diferencia demasiado sutil que se supera con un adecuado ajuste ya sea en la tasa o en los flujos.

Para mayor utilidad de los diferentes empresarios que podrían ser lectores de este artículo, se ha elaborado una tabla para el modelo CAPM que incluye los diferentes sectores para los cuales Damodaran calcula el correspondiente Beta y se ha calculado el costo de capital para varios niveles de la razón Deuda/Patrimonio (20\%, $30 \%, 40 \%$ y $50 \%$ ) disponibles en la tabla 11 .

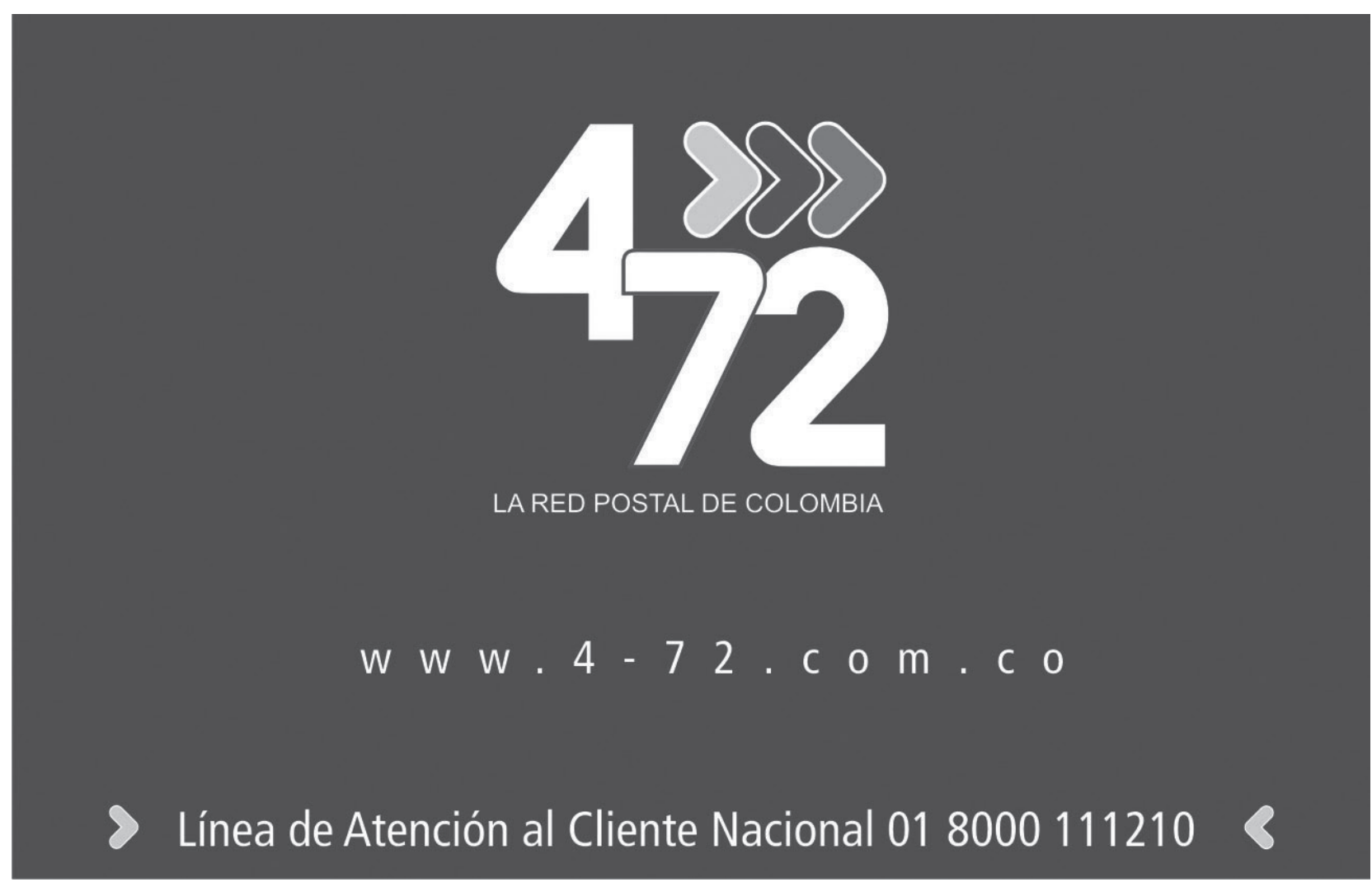


Tabla 11. Costo de capital en Colombia para varios sectores t. razón $\mathbf{D} / \mathbf{P}$

\begin{tabular}{|c|c|c|c|c|c|}
\hline \multirow{3}{*}{ Sector } & \multirow{3}{*}{$\begin{array}{l}\text { Beta no a } \\
\text { apalancado }\end{array}$} & \multicolumn{4}{|c|}{ RAZON D/P } \\
\hline & & $20,00 \%$ & $30,00 \%$ & $40,00 \%$ & $50,00 \%$ \\
\hline & & \multicolumn{4}{|c|}{ COSTO DE CAPITAL } \\
\hline Investment Co. & $0,4624 \%$ & 11,881 & 12,016 & $12,151 \%$ & $12,286 \%$ \\
\hline Machinery & $0,6308 \%$ & 11,979 & $12,163 \%$ & $12,348 \%$ & 12,532 \\
\hline Metals \& $\mathrm{M}$ in ing & $0,7492 \%$ & 12,048 & 12,267 & $12,486 \%$ & 12.704 \\
\hline Oficce Equipment \& Services & $0,4520 \%$ & 11,875 & 12,007 & $12,139 \%$ & 12,271 \\
\hline Oil/Gas (Integrated) & $0,7276 \%$ & $12,036 \%$ & $12,248 \%$ & $12,461 \%$ & $12,673 \%$ \\
\hline Oil/Gas (Prodution and Exploration) & $0,8924 \%$ & $12,132 \%$ & $12,393 \%$ & $12,653 \%$ & $12,914 \%$ \\
\hline Oil/Gas Distribution & $0,5029 \%$ & $11,905 \%$ & $12,051 \%$ & $12,198 \%$ & $12,345 \%$ \\
\hline Oilfield Svcs/Equip. & $0,6312 \%$ & $11,979 \%$ & $12,164 \%$ & $12,348 \%$ & $12,532 \%$ \\
\hline Packaging \& Container & $0,4009 \%$ & $11,845 \%$ & $11,962 \%$ & $12,079 \%$ & $12,196 \%$ \\
\hline Paper/Forest Products & $0,4253 \%$ & $11,859 \%$ & $11,983 \%$ & $12,108 \%$ & $12,232 \%$ \\
\hline Pharma \& Drugs & $0,5371 \%$ & $11,924 \%$ & $12,081 \%$ & $12,238 \%$ & $12,395 \%$ \\
\hline Power & $0,3866 \%$ & $11,837 \%$ & $11,949 \%$ & $12,062 \%$ & $12,175 \%$ \\
\hline Precious Metal & $0,8461 \%$ & $12,105 \%$ & $12,352 \%$ & $12,599 \%$ & $12,846 \%$ \\
\hline Publshing \& Newspapers & $0.4859 \%$ & $11,895 \%$ & $12,036 \%$ & $12,178 \%$ & $12,320 \%$ \\
\hline R.E.I.T. & $0,4324 \%$ & $11,863 \%$ & $11,990 \%$ & $12,116 \%$ & $12,242 \%$ \\
\hline Railroad & $0,5186 \%$ & $11,914 \%$ & $12,065 \%$ & $12,217 \%$ & $12,368 \%$ \\
\hline Real Estate & $0,5453 \%$ & $11,929 \%$ & $12,088 \%$ & $12,248 \%$ & $12,407 \%$ \\
\hline Real Estate (Development) & $0,6317 \%$ & $11,980 \%$ & $12,164 \%$ & $12,349 \%$ & $12,533 \%$ \\
\hline Real Estate (Operations \& Services) & $0,3955 \%$ & $11,842 \%$ & $11,957 \%$ & $12,073 \%$ & $12,188 \%$ \\
\hline Recreation & $0,5479 \%$ & $11,931 \%$ & $12,091 \%$ & $12,251 \%$ & $12,411 \%$ \\
\hline Reinsurance & $0,7460 \%$ & $12,046 \%$ & $12,264 \%$ & $12,482 \%$ & $12,700 \%$ \\
\hline Restaurant & $0,4763 \%$ & $11,889 \%$ & $12,028 \%$ & $12,167 \%$ & $12,306 \%$ \\
\hline Retail (Automotive) & $0,3211 \%$ & $11,798 \%$ & $11,892 \%$ & $11,986 \%$ & $12,080 \%$ \\
\hline Retail ( Building Supply) & $0,4529 \%$ & $11,875 \%$ & $12,008 \%$ & $12,140 \%$ & $12,272 \%$ \\
\hline Retail (Distributors) & $0,5262 \%$ & $11,918 \%$ & $12,072 \%$ & $12,225 \%$ & $12,379 \%$ \\
\hline Retail (General) & $0,5599 \%$ & $11,938 \%$ & $12,101 \%$ & $12,265 \%$ & $12,428 \%$ \\
\hline Retail (Grocery and Food) & $0,4368 \%$ & $11,866 \%$ & $11,993 \%$ & $12,121 \%$ & $12,249 \%$ \\
\hline Retail (Internet) & $0,9724 \%$ & $12,179 \%$ & $12,463 \%$ & $12,746 \%$ & $13,030 \%$ \\
\hline Retail (Special Lines) & $0,5953 \%$ & $11,958 \%$ & $12,132 \%$ & $12,306 \%$ & $12,480 \%$ \\
\hline Rubber \& Tires & $0,5142 \%$ & $11,911 \%$ & $12,061 \%$ & $12,211 \%$ & $12,361 \%$ \\
\hline Semiconductor & $0,6427 \%$ & $11,986 \%$ & $12,174 \%$ & $12,361 \%$ & $12,549 \%$ \\
\hline Semiconductor Equip & $0,7223 \%$ & $12,033 \%$ & $12,244 \%$ & $12,454 \%$ & $12,665 \%$ \\
\hline Shipbuilding \& Marine & $0,5797 \%$ & $11,949 \%$ & $12,119 \%$ & $12,288 \%$ & $12,457 \%$ \\
\hline Shoe & $0,7044 \%$ & $12,022 \%$ & $12,228 \%$ & $12,433 \%$ & $12,639 \%$ \\
\hline Steel & $0,6935 \%$ & $12,016 \%$ & $12,218 \%$ & $12,421 \%$ & $12,623 \%$ \\
\hline Telecom (Wireless) & $0,5988 \%$ & $11,961 \%$ & $12,135 \%$ & $12,310 \%$ & $12,485 \%$ \\
\hline Telecom. Equipment & $0,7402 \%$ & $12,043 \%$ & $12,259 \%$ & $12,475 \%$ & $12,691 \%$ \\
\hline Telecom.Services & $0,4940 \%$ & $11,899 \%$ & $12,044 \%$ & $12,188 \%$ & $12,332 \%$ \\
\hline Thrift & $0,7410 \%$ & $12,044 \%$ & $12,260 \%$ & $12,476 \%$ & $12,693 \%$ \\
\hline Tobacco & $0,3300 \%$ & $11,804 \%$ & $11,900 \%$ & $11,996 \%$ & $12,093 \%$ \\
\hline Tranportation & $0,4295 \%$ & $11,862 \%$ & $11,987 \%$ & $12,112 \%$ & $12,238 \%$ \\
\hline Trucking & $0,3939 \%$ & $11,841 \%$ & $11,956 \%$ & $12,071 \%$ & $12,186 \%$ \\
\hline Utility (General) & $0,0918 \%$ & $11,664 \%$ & $11,691 \%$ & $11,718 \%$ & $11,745 \%$ \\
\hline Utility (Water) & $0,4073 \%$ & $11,849 \%$ & $11,968 \%$ & $12,086 \%$ & $12,205 \%$ \\
\hline Total Market & $0,5647 \%$ & $11,941 \%$ & $12,105 \%$ & $12,270 \%$ & $12,435 \%$ \\
\hline
\end{tabular}

Fuente. Sectores y betas: Damodaran on line. Levered and unleveres betas by industry. Disponible en http://pages.stern.nyu.edu/ adamodar/. Consulta:25 de febrero de 2010. Costo de Capital: cálculos propios. 


\section{CONCLUSIONES}

De los tres modelos enunciados, se han aplicado los dos que se basan en el modelo CAPM (Capital Assets Pricing Model por sus siglas en inglés o modelo de valuación de activos de capital) y sus autores aportan una extensión del modelo como solución al cálculo del costo de capital para países emergentes. Se ha seleccionado de una parte el modelo de Damodaran que incluye un ajuste mediante el cálculo de la prima de riesgo país, country equity risk Premium. De otra parte está el modelo de Godfrey y Espinosa que proponen el ajuste acorde con la relación entre el mercado en Estados Unidos y el mercado del país emergente.

Los dos modelos representan una aproximación al costo de Equity o Patrimonio y logran un acercamiento teórico interesante para medir la relación riesgo y rendimiento de una compañía frente al mercado. Su estimación para países emergentes se hace más complejo dados factores estructurales de estos mercados como lo son su alta volatilidad, la poca profundización de sus mercados de capitales, los datos históricos insuficientes de sus mercados financieros, la dificultad de establecer el grado de interdependencia con los mercados internacionales y en general sus políticas macroeconómicas que le agregan mayor riesgo país.

El uso de metodologías de aproximación al cálculo del costo de inversión en países emergentes busca superar estos obstáculos y cuantificar el riesgo adicional que implica traspasar la frontera de mercados maduros, como lo es el de Estados Unidos o cualquier país desarrollado y medir las implicaciones que tiene la ubicación de activos por parte de un inversionista en estos mercados. El punto de partida de las dos metodologías seleccionadas es el CAPM, calculado inicialmente para el mercado americano, tiene un profundo referente en la teoría de portafolios.

La decisión en el uso de una de las tasas por parte de una empresa determinada dependerá de su propio perfil de riesgo y de las perspectivas económicas que tenga. Algunos elementos como el tipo de cambio, los ajustes en la volatilidad y en los betas, siguen teniendo un componente subjetivo que debe ser analizado en el momento de la aplicación de la tasa de descuento obtenida.

La tasa sugerida es la del modelo de Damodaran que incluye el beta específico de la industria para países emergentes por lo que se considera es más preciso que el modelo de Godfrey y Espinosa que llega hasta prima de riesgo país.

Para quien requiera estimar la tasa de descuento de una compañía colombiana con operación internacional en diferentes países, debe efectuar una metodología similar con la información de mercado de cada país. En la estimación de la tasa de descuento corporativa para la misma compañía sería necesario analizar la operación global, incluyendo diversos valores de $\lambda$ (lamda) para cada país en el cual opera, de acuerdo con algún criterio relacionado con la generación de ingresos, o nivel de exportaciones entre otros. La tasa de descuento esperada estaría compuesta por:

Expected Return $=\mathbf{R f}+$ Beta $($ Mature Equity Risk Premium) $+\lambda$ (County Risk Premium)

Para lograrlo es necesario disponer de la información financiera y operativa global de la compañía y el análisis del comportamiento del país en cuestión similar a como lo hemos efectuado para el caso colombiano.

La tabla 11 le permite a empresarios que no tienen familiaridad con el tema, referenciar el costo de capital de su empresa ubicando el sector, su razón D/P aproximada particular de cada empresa - y obtener el costo de capital. 


\section{BIBLIOGRAFíA}

Aswath, D. (1999), Equity risk premium, Stern School of Business, 27.

Erb, C. B; Harvey, C. et al (1996). Political risk, economic risk, and financial risk, Financial Analysts Journal; ABI/INFORM Global, 52, 6.

Estrada, J. (2000). The cost of equity in emerging markets: A downside risk approach. IESE, Barcelona. August.

Ian A., et al (1996). Regulatory structure and risk and infrastructure firms. An international comparison. The World Bank, Private Sector Development Department, December.

Información Financiera compañía sector alimentos, Superintendencia de Sociedades, Colombia. Disponible en http://sirem.supersociedades.gov.co/SIREM/index.jsp. Consulta: 25 de febrero de 2010.

Lintner, J. (1965). The Valuation of Risk Assets and The Selection of Risky Investments in Stock Portfolios and Capital Budgets, Review of Economics and Statistics Vol. 47: 13-37

Mossin,J. Equilibrium in a Capital Asset Market, Econometría 34 pp. 768-783.

Sharpe,W. (1964). Capital Asset Prices: A Theory of Market Equilibrium under Conditions of Risk, Journal of Finance. 425-442

Stephen G., y Espinosa R. (1996) "A practical approach to calculating Cost of Equity for investment in Emerging Markets." Journal of Applied Corporate Finance, fall .volume 9, 80-89.

Treynor, J. (1961). Toward a Theory of the Market Value of Risk Assets, manuscrito no publicado. 


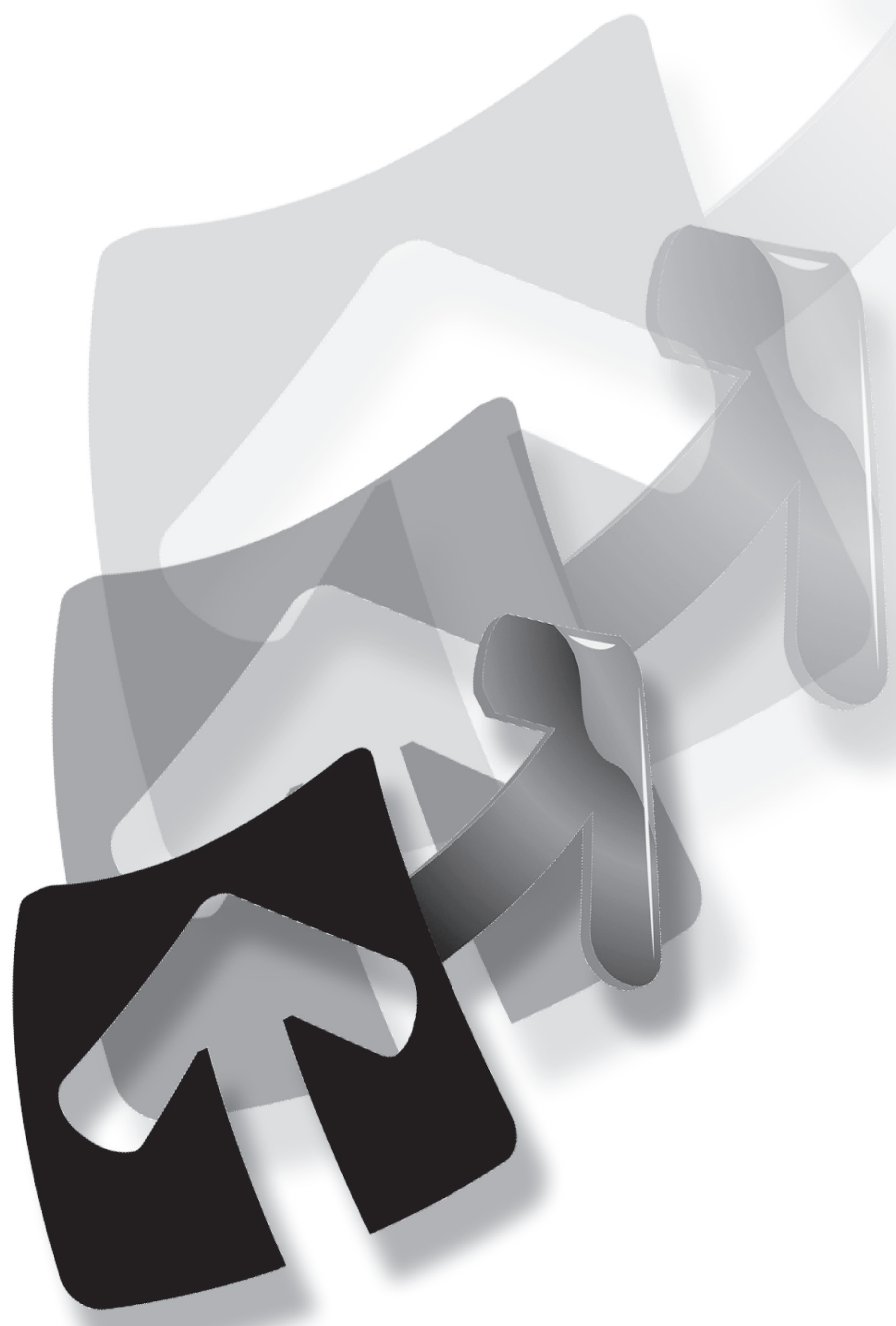

